\title{
Cortico-limbic-striatal circuits subserving different forms of cost-benefit decision making
}

\author{
Stan B. Floresco, Jennifer R. St. Onge, \\ SARVIn GHOdS-Sharifi, AND CATHARINE A. Winstanley \\ University of British Columbia, Vancouver, British Columbia, Canada
}

\begin{abstract}
Research on the neural basis that underlies decision making in humans has revealed that these processes are mediated by distributed neural networks that incorporate different regions of the frontal lobes, the amygdala, the ventral striatum, and the dopamine system. In the present article, we review recent studies in rodents investigating the contribution of these systems to different forms of cost-benefit decision making and focus on evaluations related to delays, effort, or risks associated with certain rewards. Anatomically distinct regions of the medial and orbital prefrontal cortex make dissociable contributions to different forms of decision making, although lesions of these regions can induce variable effects, depending on the type of tasks used to assess these functions. The basolateral amygdala and the nucleus accumbens play a more fundamental role in these evaluations, helping an organism overcome different costs to obtain better rewards. Dopamine activity biases behavior toward more costly yet larger rewards, although abnormal increases in dopamine transmission can exert opposing actions on different types of decision making. The fact that similar neural circuits are recruited to solve these types of problems in both humans and animals suggests that animal models of decision making will prove useful in elucidating the mechanisms mediating these processes.
\end{abstract}

Since the pioneering work of Damasio and colleagues, there has been growing interest in the neuropsychological basis of decision making. In the laboratory, this form of executive functioning has been assessed with different types of "gambling" tasks that are designed to simulate real-life decisions in terms of uncertainty, reward, and punishment. In the original version (now referred to as the "Iowa Gambling Task"), subjects choose between decks of cards that yield either high immediate monetary gain but larger future loss, or smaller gains and smaller future loss. Normal subjects eventually learn the optimal strategy, selecting from the low-risk decks to obtain long-term gains. In their original studies, Bechara, Damasio, and colleagues observed that patients with damage to the ventromedial regions of the prefrontal cortex (PFC) - encompassing the orbitofrontal cortex (OFC) and ventral aspects of the anterior cingulatedisplayed impaired decision making, making more high-risk choices (Bechara, Damasio, Damasio, \& Anderson, 1994; Bechara, Damasio, Damasio, \& Lee, 1999). These initial findings spawned a marked increase in research attempting to elucidate neural circuits that mediate decision making. With the development of novel tasks, assessments of patient populations, and the exploitation of functional brain imaging, cognitive neuroscience has made notable progress in identifying some of the distributed neural circuits that mediate different aspects of cost-benefit decision making.

In addition to ventromedial and orbital regions, damage to the dorsolateral PFC or the dorsomedial sectors of the anterior cingulate has also been associated with impaired performance on different types of tasks requiring cost-benefit evaluations about risks and rewards (Clark, Manes, Antoun, Sahakian, \& Robbins, 2003; Fellows \& Farah, 2005; Manes et al., 2002; Rogers et al., 1999). In addition, the notion that the OFC plays a critical role in specifically mediating risk-based decisions has been tempered by more recent findings. For example, in the Iowa Gambling Task, the probability that choice from a risky deck will yield net gain or reward is not random; instead, the deck is biased so that subjects are rewarded more rather than punished early in the session, thus "tempting" the subject to favor the risky deck. As the session progresses, the subject is exposed to an increasing number of large punishments, and healthy controls begin to switch their preferences to the advantageous decks. However, if the order of gains and losses is randomized, OFC patients perform similarly to controls (Fellows \& Farah, 2005). On the Cambridge Gambling Task, in which subjects are explicitly informed of the odds associated with a choice, OFC patients sometimes show normal decision making and can actually display risk-averse tendencies (Manes et al., 2002; Rogers et al., 1999). Accordingly, impairments in decision making induced by OFC lesions may be attributable to an inability to shift choice toward the low-risk decks after initial exposure to large rewards associated with high-risk decks (Dalley, Cardinal \& Robbins, 2004; Fellows, 2007; Ragozzino, 2007). It is also important that one note that 
there have been considerable discrepancies between studies in terms of which regions of the PFC play a critical role in these decision-making processes (Bechara et al., 1999; Bechara, Damasio, Tranel, \& Anderson, 1998; Clark et al., 2003; Fellows \& Farah, 2005; Manes et al., 2002; Rogers et al., 1999). It is therefore apparent that some regions of the frontal lobes mediate these processes, but the specific cognitive operations processed by different subregions of the PFC that facilitate these decisions remain unclear.

The amygdala - a temporal lobe structure reciprocally connected with the medial PFC and OFC - has also been implicated in mediating different aspects of decision making. Patients with damage to this region consistently display impaired decision making, making more disadvantageous, "risky" choices on a number of different tasks (Bechara et al., 1999; Brand, Grabenhorst, Starcke, Vandekerckhove, \& Markowitsch, 2007). Unlike in patients with PFC damage, increased risky choices observed in patients with amygdala damage may be specific to decisions involving potential gains but not potential losses (Weller, Levin, Shiv, \& Bechara, 2007). Thus, it has been proposed that the amygdala contributes to evaluations of the potential rewards associated with different options, whereas as-ofyet undetermined region(s) of the PFC integrate cognitive and emotional information about potential gains and losses to determine an appropriate course of action (O'Doherty, 2004; Weller et al., 2007).

Functional imaging studies in normal subjects have provided further insight into the neural networks that mediate these processes. In keeping with findings from braindamaged patients, tasks requiring cost-benefit evaluations about risk and rewards activate distributed networks that include the $\mathrm{OFC}$, medial/cingulate PFC, and amygdala (Blair et al., 2006; Marsh, Blair, Vythilingam, Busis, \& Blair, 2007; Rogers et al., 2004). However, given the correlative nature of these studies, controversies remain about what the activation of these areas actually reflects (e.g., input vs. output to/from a particular region), as well as the specific cognitive contributions made by these regions. For example, activation of the anterior cingulate has been proposed to be related to the resolution of response conflict (Rogers et al., 1999), evaluations of risks (Paulus \& Frank, 2006), punishments (Blair et al., 2006), or expected rewards (Marsh et al., 2007). However, imaging studies have been useful in identifying certain subcortical structures (i.e., those typically not damaged in human patients) that may also contribute to these processes. Specifically, the activation of the nucleus accumbens (NAc) has consistently been correlated in a number of different decision-making paradigms with making risky choices (Kuhnen \& Knutson, 2005; Tom, Fox, Trepel, \& Poldrack, 2007). This region of the ventral striatum receives input from the medial $\mathrm{PFC}$ and the ventromedial and lateral regions of the OFC (Reynolds \& Zahm, 2005), as well as the amygdala, making this nucleus ideally situated to integrate information from different limbic-cortical regions to guide response selection (Floresco, 2007). In a similar vein, the activation of the ventral striatum has been shown to be positively correlated in a delay-discounting paradigm with an increased preference for smaller immediate over larger delayed rewards (Hariri et al., 2006). These studies indicate that in ambiguous situations requiring judgments about the different costs and magnitudes of rewards associated with different actions, the selection of a particular action is mediated by distributed neural circuits that incorporate different regions of the frontal lobes, the amygdala, and the ventral striatum.

It is notable that all of the above-mentioned brain regions receive a dense dopaminergic input from the ventral tegmental area. This anatomical arrangement suggests that perturbations in dopamine (DA) transmission may hamper decision-making processes - an assertion supported by studies of neuropsychiatric patients where alterations in the DA system are thought to contribute to their disorders. Patients with schizophrenia (Hutton et al., 2002; Shurman, Horan, \& Nuechterlein, 2005), Parkinson's disease (Pagonabarraga et al., 2007), and depression (Must et al., 2006) and stimulant abusers (Rogers et al., 1999) all display abnormal choice behavior when challenged with a number of decision-making tasks. Furthermore, both schizophrenics and stimulant abusers display patterns of impulsive choice on delay-based decision-making tasks; they prefer smaller, hypothetical rewards over larger rewards that would be received after a considerable delay (Heerey, Robinson, McMahon, \& Gold, 2007; Monterosso, Ehrman, Napier, O'Brien, \& Childress, 2001). Note that many of these studies used medicated patients, and some studies have indicated that increases or decreases in DA activity induced by these treatments may contribute to alterations in decision making (Cools, Barker, Sahakian, \& Robbins, 2003; Roiser et al., 2005; Swainson et al., 2000). These studies provide indirect evidence that mesocorticolimbic DA activity makes an important contribution to different forms of cost-benefit decisions, although the specific cortical and subcortical areas where DA may be acting to influence these decisions remains unclear.

It is clear from the above-mentioned findings that choosing between rewards associated with different costs or punishments is dependent on transcortical networks incorporating different regions of the $\mathrm{PFC}$, the amygdala, the ventral striatum, and the DA system. Yet, the restrictions associated with human research limit the ability to ascertain the specific contributions that these regions make to cognitive operations related to these processes. Recent work using animal models of cost-benefit decision making has provided important information about the contribution that these interconnected neural systems make to different components of decision making. However, certain considerations must be taken into account when attempting to assess decision making in rodents. In human decision-making paradigms, "punishment" is typically the loss of a previously obtained monetary reward, whereas most rodent tasks use primary reinforcement (e.g., food) as rewarding stimuli. Simply put, it is difficult to take away a reward from a rat that has already consumed its food pellet. Nevertheless, a key component of decision making that can be assessed in rodents is the evaluation of costs associated with different candidate actions as compared with the potential rewards that may be obtained by those actions. In these studies, animals choose between response options that yield either a smaller reward that 
comes with a nominal response cost, or a larger or more palatable reward that comes with a greater cost. For the purposes of this review, the relative response "cost" is defined as an experimental manipulation associated with one response option that somehow impedes access to a larger or more-preferred reward. All things being equal, animals typically choose more versus less food; however, the imposition of certain costs leads to a "discounting" of larger rewards. Three such costs that are particularly effective in biasing choice behavior in animals are (1) delaying the delivery of the reward, (2) requiring animals to exert greater physical effort to obtain the reward, or (3) making the delivery of the reward probabilistic (i.e., uncertain/ risky). On the surface, the latter two manipulations may seem to be special cases of delayed reinforcement. However, as will be described, it appears that there are distinct differences in the neural circuits that are recruited to solve these different types of decision-making problems. Thus, the main focus of this review will be to highlight some recent studies assessing the neural basis of delay, effort, and risk-based decision making in rodents, focusing on the contributions that the PFC, amygdala, NAc, and DA system make to these processes. Since each of these systems has been shown to play a role in decision making in humans, the overarching theme of this review will be to draw insight from work with experimental animals into the specific contributions that these systems make in resolving cost-benefit evaluations in humans.

\section{Delay-Based Decision Making}

The central principle underlying delay-discounting paradigms is that subjects are trained to choose between a small reward delivered immediately versus a larger reward delivered after a delay. As in humans, the selection of the small, immediate reward over the larger, delayed reward is thought to reflect a type of impulsive decision making.

Although the concept seems relatively simple, numerous different paradigms have been developed. One of the most prevalent is that first described by Evenden and Ryan (1996): Animals choose to respond on two levers, one of which provides a small reward of one pellet, the other a large reward of four pellets. The length of each trial is kept constant so that selection of the larger reward option is always the optimal strategy in terms of obtaining the maximal number of reward pellets per session. In successive blocks of trials, the delay to the large reward increases, and animals shift their preference from the large to the smaller reward. This can be contrasted with simpler tests using a T-maze, in which the animal learns that one arm is baited with a larger reward, and the other with a smaller reward. If the rat chooses the large reward arm, it can be detained within the arm by the closing of the two gates. After a certain delay, the gate in front of the food is raised, and the animal has access to the reward. Typically, once the animal has learned to choose the arm associated with the larger reward, it will next learn to discriminate between an immediate reward in one arm versus a larger reward for which it must endure a relatively short delay-for example, 5-10 sec (Bizot, Le Bihan, Puech, Hamon, \& Thiebot, 1999; Rudebeck, Walton, Smyth, Bannerman, \& Rushworth, 2006). The delay to the large reward is kept constant during each daily set of trials or session; therefore, there is a between-session rather than a within-session shift in delay.

Both of these tasks can be described as "systematic"; the experimenter varies the delay to different-sized reinforcers. "Adjusting" tasks have also been developed, in which the behavior of the subject determines the length of the delays. In the adjusting-delay procedure, choice of the large reward leads to an increase in the delay to the large reward, whereas choice of the small reward leads to a decrease in the delay to the large reward (Mazur, 1987). The adjusting-amount procedure developed by Richards has a similar theoretical basis, but the delays stay constant within a session, whereas the size of the delayed reinforcer varies, depending on the choice in the previous trial (Richards, Mitchell, de Wit, \& Seiden, 1997). Eventually, an indifference point is reached; the animal chooses the large reward $50 \%$ of the time. This point is used as a measure of impulsivity (but see Cardinal, Daw, Robbins, \& Everitt, 2002).

Such methodological divergence has, perhaps unsurprisingly, led to some discrepancies concerning the effects of particular lesions or pharmacological manipulations on choice, and different tasks may vary in the extent to which different areas or neurotransmitter systems are recruited. In most cases, such findings have stimulated further experiments and ultimately improved our understanding of the factors that influence delay-discounting behavior.

Prefrontal cortex. Despite the well-documented role for the anterior cingulate region of the medial PFC in conflict resolution (Barch et al., 2001) and the exertion of mental effort (Naccache et al., 2005), selective excitotoxic lesions of this region do not affect delaydiscounting performance in rats, regardless of which type of delay-discounting paradigm is used (Cardinal, Pennicott, Sugathapala, Robbins, \& Everitt, 2001; Rudebeck et al., 2006). Damage to the more ventromedial prelimbic cortex did alter choice behavior, but led to a flattening of the delay-discounting curve rather than to a shift in preference toward more immediate or more delayed rewards (Cardinal et al., 2001). Rats with prelimbic cortex lesions chose the larger reward less, early in the session, when the delay was short, but more often, later in the session, when the delay increased. Rather than interpreting this choice pattern as being an increase or decrease in impulsive decision making, it seems more plausible that the lesion may have rendered the rat insensitive to within-session shifts in contingencies. Hence, these animals appear to average their responding on the large reward lever across the session, regardless of delay. The prelimbic cortex, in particular, has a pronounced role in the detection of instrumental contingencies (Balleine \& Dickinson, 1998), and delay to reinforcement certainly impairs the detection of a causal contingency between response and reward delivery (Lattal \& Gleeson, 1990). Alternatively, the frontal lesion could have impaired temporal judgments so that the rats were unable to use temporal cues to estimate how much time had passed since the session started, and therefore which contingency was in play. Although forced choice trials are included in the experiment to "remind" the rat of the current delay-reward trade-off, internally driven temporal 
judgments may also play a role. Damage to the prelimbic cortex can impair aspects of time perception (Dietrich \& Allen, 1998). In order to resolve whether the prelimbic cortex is actually involved in decision-making processes that are pertinent to delay discounting as opposed to the detection of temporal signals, it would be useful for one to investigate the effects of lesions of this region of the PFC on tests that use a between-sessions rather than a withinsession shift in delay.

Perhaps the frontal region that has received the most attention with respect to its role in delay-based decision making is the OFC. A decreased preference for larger, delayed rewards was observed in a two-lever choice task using a between-sessions shift in delay following lesions to the OFC (Mobini et al., 2002). However, the opposite effect was reported when a within-session shift in delay was used, with animals showing a marked preference for larger delayed rewards (Winstanley, Theobald, Cardinal, $\&$ Robbins, 2004). One key difference between the two experiments was that in the former, the lesions were made before animals had acquired the task, whereas in the latter, lesions were made subsequent to the acquisition of stable baseline behavior. Recent data, however, suggest that this difference is not sufficient to explain these discrepant results. Rudebeck et al. (2006) observed that damage to the OFC that was induced after task acquisition significantly, but temporarily, increased the choice of the smaller, immediate reward. This experiment used a maze-based test in which the length of the delay to the large reward was shifted between sessions (as in Mobini et al., 2002), and choice of the large delayed reward was very high $(\sim 85 \%)$. Could the effect of OFC lesions be determined by whether rats have to shift their preference within or between sessions, or are other factors at play?

Considering both tasks, there may be cues present in the maze-based task that could signal choice of the delayed reward, but that are not as apparent in the tasks conducted in the operant chambers. For example, the closing of the gates signals the start of the delay, and the lifting of the gate signals reward delivery. Also, the baseline level of impulsive choice may influence the effects of OFC damage. Preliminary findings indicate that the ability of the inactivation of the OFC to increase or decrease impulsive choice can indeed be affected by such factors (Zeeb, Floresco, \& Winstanley, 2007). These experiments used a within-session shift in delay, as per the original Evenden and Ryan task (1996). For one set of rats, the delay to the large reward was signaled by illumination of a cue light located above the large reward lever, whereas in another group, no such cue was presented during the delay period (i.e., the chamber remained in darkness; Cardinal, Robbins, \& Everitt, 2000). For animals in the cued condition, the cue light was illuminated once the larger, delayed reward was chosen, and it remained illuminated until the larger reward was delivered. As such, the cue light bridged the delay and signaled that reward would (eventually) be delivered, and may therefore facilitate choice of the delayed reward by acting as a conditioned reinforcer (see Cardinal et al., 2000, for further discussion). Using a cue speeded the acquisition of the task, but the general level of impulsivity shown by the cued and uncued groups was comparable. Inhibiting activity in the OFC via infusions of GABA agonists significantly decreased impulsive choice if the delay was not cued, reproducing previous findings using this task (Winstanley et al., 2004). In contrast, OFC inactivation increased impulsive choice if the delay was cued, but only in animals that showed a low level of impulsive choice at baseline - that is, there was a significant interaction between the basal level of impulsivity and the effects of the cortical inactivation. These findings mimic many aspects of Rudebeck et al. (2006) and suggest that the extent to which cues signaling delivery of the large reward are present during a delay has marked effects on the contribution of the OFC to this form of decision making. It is also worth noting that rats in the Rudebeck et al. (2006) study received fewer trials prior to lesioning than did animals in the Winstanley et al. (2004) study, due to the laborintensive nature of maze-based training. This circumstance may also contribute to the low level of impulsive choice in the former study, and ultimately to the difference in the OFC lesions' effect. However, data from this cued operant task - in which training was more extensive-indicate that the simple difference in the number of training trials is unlikely to fully explain the discrepant findings. Given the widespread use of cues in gambling, this issue clearly warrants further investigation.

When the delay is uncued, the finding that increased choice of the large reward can be induced by damage or inactivation of the $\mathrm{OFC}$ also requires explanation. Although seemingly paradoxical, this persistent choice of the large reward despite its associated aversive consequences (i.e., the delay) could reflect a "myopia for the future" comparable to that reported in human patients with OFC damage on laboratory-based gambling tasks (Bechara et al., 1994). Thus, damage to the OFC may prevent the adequate integration of information about the consequences of responding for a reward with the subjective value of that rewarding outcome (Schoenbaum, Chiba, \& Gallagher, 1999), so that delay fails to sufficiently devalue the larger reward. The OFC has a well-established role in updating the internal representation of value of a reward when that value changes - a process that is taxed more heavily in delaydiscounting paradigms incorporating a within-session shift in delay. Alternatively, the increased choice of the large reward could reflect perseverative behavior that has repeatedly been associated with OFC lesions (Boulougouris, Dalley, \& Robbins, 2007; Chudasama et al., 2003; Chudasama \& Robbins, 2003). Exploring the role of the OFC in delay-discounting behavior has certainly highlighted the complexity involved in such a conceptually simple decision process. However, work of this kind can only increase our understanding of the nature of information processing inherent within delay discounting.

Amygdala. The basolateral amygdala shares many reciprocal connections with different regions of the PFC, and a functional connection between these two regions is important for aspects of goal-directed behavior (Baxter, Parker, Lindner, Izquierdo, \& Murray, 2000). Despite the fact that the OFC is a higher order region of association neocortex and the basolateral amygdala is a subcortical 
nucleus within the highly conserved limbic system, it has been difficult to functionally dissociate the contributions of these two areas, because lesions to the OFC and basolateral amygdala often cause similar behavioral effects (Schoenbaum, Setlow, \& Ramus, 2003). However, in contrast with the effects of OFC lesions, the destruction of the basolateral amygdala increased choice of the small, immediate reward on the same task (Winstanley et al., 2004). This increase in impulsive choice may be explained as an impairment in utilizing representations of the incentive value of reward to guide behavior. In order to maintain responding for the large reward when it is delayed, subjects must theoretically hold a representation of the reward "online" and anticipate its delivery during the delay period. Basolateral amygdala lesions may prevent the utilization of such representations so that the value of the large reward is not maintained across the delay. This explanation is consistent with the important role of the basolateral amygdala in mediating conditioned reinforcement processes. Although no explicit conditioned reinforcers or cues signaling the delay were present in the task, responding on the lever leading to delivery of the large rewardor the postresponse delay itself - may function as implicit conditioned reinforcers and help to maintain responding (Garrud, Goodall, \& Mackintosh, 1981).

Nucleus accumbens. Similar to the effects of lesions of the basolateral amygdala, a dramatic increase in choice of the small, immediate reward has been observed in rats following excitotoxic lesions to the lateral core region of the NAc, using a delay-discounting task that employed within-session shifts in the delay to the large reward (Cardinal et al., 2001). However, a recent article has reported that lesions to the NAc have very different effects on delay discounting, as assessed using an adjusting-amount method (Acheson et al., 2006). In both studies, the delay to the large reward was not signaled by a cue light or any other explicit stimulus. In the latter study, all rats reached similar indifference points, indicating that both lesioned and sham-treated rats were discounting to the same degree. When the delay to the large reward was systematically varied between days to produce a delay-discounting curve, NAc-lesioned rats were less sensitive to delay increases. The authors of this study suggest that this insensitivity to changes in the delay to reinforcement could give rise to behavior that appears more or less impulsive, depending on the way the delay-discounting task is structured. Although this is an interesting hypothesis, it is also worth noting that both the NAc core and shell were damaged in the Acheson et al. (2006) study, whereas Cardinal et al. (2001) only targeted the core. These two areas have very distinct functions in mediating reward-related learning, and damage to both regions may well result in a different pattern of behavior than damage to only one or the other in isolation. The effects of selective lesions to the NAc shell on delaydiscounting performance may help resolve these issues.

Dopamine. The majority of studies have shown that systemic administration of psychostimulants, such as amphetamine, increases the choice of larger delayed rewards, in parallel to their clinical effects on impulsive behaviors (Floresco, Tse, \& Ghods-Sharifi, 2008; van Gaalen, van
Koten, Schoffelmeer, \& Vanderschuren, 2006; Wade, de Wit, \& Richards, 2000; Winstanley, Theobald, Dalley, \& Robbins, 2003, 2005), although some studies have shown the opposite effect, depending on a number of factors, such as dose and specific experimental conditions (Cardinal et al., 2000; Evenden \& Ryan, 1996). The ability of these drugs to bias choice toward larger, delayed rewards has been largely attributed to their ability to potentiate the actions of the dopaminergic system. Administration of the $\mathrm{DA} \mathrm{D}_{2}$ receptor antagonist, eticlopride, attenuates the effects of amphetamine on delay discounting, highlighting the importance of dopamine in the effects of the drug (van Gaalen et al., 2006). $\mathrm{D}_{2}$ receptor blockade alone, however, has no effects of impulsive choice. In contrast, the $\mathrm{D}_{1}$ receptor antagonist, SCH23390, increased impulsive choice when it was given in isolation, but had no effect on the actions of amphetamine on task (van Gaalen et al., 2006). In terms of where DA may be acting to modulate choice behavior, a likely candidate was the NAc, given that accumbal DA plays an important role in signaling rewardrelated information (Wise \& Rompre, 1989). However, destruction of DA terminals in the NAc via local infusions of 6-hydroxydopamine (6-OHDA) did not change baseline choice behavior, nor did it prevent amphetamine from decreasing impulsive choice (Winstanley et al., 2005). This result was somewhat surprising, since 6-OHDA lesions of the NAc have blocked the effects of amphetamine in many other behavioral paradigms, including tests of motor impulsivity (Cole \& Robbins, 1989; Kelly, Seviour, \& Iversen, 1975; Koob, Riley, Smith, \& Robbins, 1978; Robbins, Roberts, \& Koob, 1983).

Although mesoaccumbens DA seems to play a less prominent role in delay discounting, 6-OHDA lesions of the OFC decrease impulsive choice, suggesting that DA in this area makes a significant contribution to the making of delay-discounting judgments (Kheramin et al., 2004). Such a conclusion is supported by data from an in vivo microdialysis study, in which levels of DA's main metabolite, DOPAC, increased within the OFC when rats were performing a delay-discounting task (Winstanley, Theobald, Dalley, Cardinal, \& Robbins, 2006). This increase was not seen in a yoked group whose response options were predetermined by the response of a master rat performing the delay-discounting paradigm. These yoked rats did not get to choose between the two levers, but were essentially performing a forced choice version of the delay-discounting paradigm in which only one lever was presented on each trial. If the "master" rat chose the large, delayed reward on a certain trial, then the yoked rat was required to respond on the large-reward lever on the corresponding trial. Hence, this yoked group performed the same pattern of responses and received the same amount of reward at the same time as the master rat: The key difference was whether they had a choice. Likewise, OFC DOPAC levels did not alter in a second yoked group that simply received the same number of food pellets at the same time as animals performing delay-discounting judgments. These data strongly suggest that the DA utilization within the OFC of animals performing the delay-discounting paradigm is related to the decision-making process involved in the task rather than 
being a function of operant responding or reward delivery. In contrast, increased DA release was observed in the medial PFC, regardless of whether animals were performing the delay-discounting task or were in one of the yoked groups; hence, reward delivery alone is sufficient to increase DA efflux in this region. This signal could theoretically contribute to the evaluation of response-outcome contingencies - a process in which the prelimbic region of the PFC is known to be involved (see above). Viewed collectively, these findings indicate that the DA systems play a critical role in enabling animals to overcome delay costs associated with the obtainment of larger rewards.

\section{Effort-Based Decision Making}

The pioneering work of John Salamone and colleagues was the first to investigate the neural basis underlying effort-related decisions. Their original studies utilized a task conducted on a T-maze in which one arm is designated as the high-reward (HR) arm, containing a larger magnitude of reward (e.g., four reward pellets), and the other the low-reward (LR) arm, containing a smaller reward (usually two pellets). To obtain the HR, rats are required to climb over a scalable barrier placed in that arm, thereby increasing the physical effort required to obtain more food relative to the effort required in the LR arm, in which no barrier is placed. During training, rats develop a preference for the HR arm, choosing to exert more effort and climb over the barrier to obtain more food on the majority of free-choice trials (Salamone, Cousins, \& Bucher, 1994). In a complementary concurrent-choice task developed by this group, rats choose between either pressing a lever a number of times to obtain a more palatable, sweetened food-pellet reward, or consuming less palatable lab chow that is freely available (Cousins, Wei, \& Salamone, 1994; Salamone et al., 1991). Again, rats prefer to engage in the instrumental response and receive the "better" reward. These procedures were initially designed to clarify the role of mesoaccumbens DA transmission in the mediation of reward-related processes (described in detail below). However, this approach has also proved useful in delineating the roles of different cortical and limbic regions in this form of cost-benefit decision making.

Prefrontal cortex. The initial studies investigating the involvement of the PFC in effort-related decisions utilized a procedure conducted on a T-maze that was based on the procedures initially described by Salamone and colleagues (1994). Rats that had received large lesions of the medial PFC, encompassing both the anterior cingulate and prelimbic regions, displayed a pronounced reduction in the preference for the HR arm (Walton, Bannerman, \& Rushworth, 2002). These effects could not be attributed to an inability to climb the barrier, impairments in spatial discrimination, or insensitivity to larger rewards, because lesioned rats performed as well as the controls when a second identical barrier was placed in the LR arm. Subsequent studies by this group using more circumscribed lesions revealed that damage to the anterior cingulate cortex - but not to the prelimbic region of the medial PFC - altered this form of decision making in a manner similar to that observed after large PFC lesions (Rudebeck et al., 2006; Walton, Ban- nerman, Alterescu, \& Rushworth, 2003). Moreover, these effects appear to be selective to the medial PFC, since similar lesions of the OFC did not alter effort-based decision making, although they did reduce the preference for larger, delayed rewards (Rudebeck et al., 2006). The results of these studies indicate that the anterior cingulate may bias decision-making processes in situations requiring evaluations of the relative effort costs attached to a particular course of action.

It is important that we point out that the ability of anterior cingulate lesions to alter effort-based decision making occurs only under some experimental conditions. In their original study, Walton and colleagues (2002) noted that the effects of medial PFC lesions could be remediated if the ratio of reward magnitude was changed from 4:2 to 5:1. In this situation, rats that had received medial PFC lesions eventually chose the HR arm as often as controls. In a similar vein, if rats with anterior cingulate lesions were trained on an equal-effort variation of the T-maze task (i.e., with barriers placed in both arms), these animals performed similarly to controls upon subsequent testing in the onebarrier condition (Rudebeck et al., 2006). Thus, it appears that with these procedures, the effects of anterior cingulate lesions are relatively transient and can be ameliorated by either increasing the benefits associated with the more effortful response option or using extended training in which the response costs are equalized. Furthermore, lesions of the anterior cingulate did not alter decision making in a concurrent-choice procedure in which rats chose between pressing a lever for a more palatable food pellet reward or eating freely available lab chow. However, these lesions were again effective in altering decision making on a T-maze-based task (Schweimer \& Hauber, 2005). Thus, it is apparent that the anterior cingulate does not play a ubiquitous role in effort-based decisions. Rather, this region of the PFC may serve a more specialized function in biasing choice behavior in situations in which the discrepancy between the particular costs and benefit associated with different choices is relatively small (e.g.; 4 vs. 2 pellets, as compared with 5 pellets vs. 1 pellet). This notion is consistent with that proposed by Cardinal et al. (2003): The anterior cingulate of the rat plays a particularly important role in discriminating between multiple stimuli on the basis of their association with reward. Likewise, findings from imaging studies in humans have also shown that increases in task difficulty are associated with a greater activation of the anterior cingulate (Barch et al., 1997; Fu et al., 2002).

Amygdala. The basolateral amygdala plays an important role in detecting changes in the magnitude of expected rewards or incentive value of instrumental outcome (Corbit \& Balleine, 2005; Salinas, Packard, \& McGaugh, 1993). In addition, lesions of this region of the amygdala increase impulsive choice in a delay-discounting paradigm, in a manner similar to lesions of the NAc (Winstanley et al., 2004). Given these findings, and in light of the anatomical connectivity between the basolateral amygdala and the $\mathrm{PFC}$, recent studies have investigated the role of this region in effort-based decision making using a T-maze protocol similar to that described previously (Floresco \& GhodsSharifi, 2007). Reversible, bilateral inactivation of the ba- 
solateral amygdala induced by infusions of the local anesthetic bupivacaine reduced the preference for the HR arm in well-trained rats, in comparison with their performance following saline infusions. However, the discounting of the HR arm was eliminated when a second barrier was introduced in the LR arm, equating the effort requirements associated with either reward. This manipulation confirmed that the effects of basolateral amygdala inactivation on effort-based decision making were not attributable to an insensitivity to reward magnitude, spatial discrimination deficits, or general motor impairments, consistent with previous findings (Winstanley et al., 2004).

A subsequent experiment directly assessed whether this form of decision making is dependent on intact serial transmission between the basolateral amygdala and the anterior cingulate region of the PFC. This study utilized an asymmetrical disconnection procedure that combined a unilateral inactivation of the basolateral amygdala with a contralateral inactivation of the anterior cingulate. Functional disconnection of these two regions again reduced the preference for the HR arm, in a manner similar to bilateral inactivation of the amygdala or lesions of the PFC. These findings suggest that the preference for animals to exert more effort to obtain larger rewards is critically dependent on a transcortical network linking the amygdala to the frontal lobes. Although the specific contribution that each region makes to this form of decision making remains to be clarified fully, one possibility is that in these situations, increased activity of basolateral amygdala neurons may encode the expected magnitude of reward associated with different choices (Pratt \& Mizumori, 1998; Saddoris, Gallagher, \& Schoenbaum, 2005; Schoenbaum, Chiba, \& Gallagher, 1998). This reward-related information may be relayed to the PFC, which can bias behavior in a particular direction by integrating these signals with other information about the response costs associated with each action, possibly mediated via connections with motor cortices (Reep, Goodwin, \& Corwin, 1990; Sesack, Deutch, Roth, $\&$ Bunney, 1989). Once a particular course of action has been determined, the transformation of this strategy into the appropriate behavioral output is likely mediated by corticostriatal connections linking the PFC to the NAc.

Nucleus accumbens and dopamine. The original studies on the neural basis of effort-based decision making focused on the role of DA transmission, placing a particular emphasis on the role of dopaminergic activity in the NAc. The theoretical impetus for these studies was to reevaluate the role of DA in motivational and reward-related processes. Although it was been widely believed that DA plays an important role in the rewarding and hedonic aspects of natural or drug reinforcers, more recent studies have brought this view into question. Taste reactivity studies in both experimental animals and Parkinsonian patients who suffer from reduced DA activity have revealed that interference with DA transmission does not alter the palatability of particular food rewards (reviewed in Salamone, Correa, Farrar, \& Mingote, 2007). Similarly, experimental evidence suggests that reducing DA receptor activity can dampen motor responses and leave motivational behavior unaltered. In this regard, systemic administration of DA antagonists or the destruction of DA terminals in the NAc severely disrupts rats' preferences to exert more physical effort to obtain a larger or more palatable reward. Using a T-maze task, Salamone and colleagues (1994) reported that rats forced to overcome a higher cost (i.e., climbing a barrier) to obtain the larger four-pellet reward showed pronounced discounting for the HR arm following treatment with low doses of the $\mathrm{D}_{2}$ antagonist haloperidol that do not disrupt discrimination between rewards of different magnitudes. Similar effects were observed following DA lesions of the NAc in rats that had received extensive preoperative training. In contrast, rats that did not have to climb a barrier to obtain a larger reward behaved in a manner similar to that of vehicle-treated rats. In addition, these manipulations reduced the preference to press a lever for a more palatable food pellet reward, with rats instead consuming more of the less preferred but freely available lab chow on a concurrent-choice task (Cousins et al., 1994). Subsequent studies by this group have shown that within the NAc, DA appears to be acting on both $\mathrm{D}_{1}$ and $\mathrm{D}_{2}$ receptors to mediate effort-based decisions, since the local blockade of either of these receptors also shifts behavior away from leverpressing for reward pellets and increases the consumption of lab chow (Cousins et al., 1994; Nowend, Arizzi, Carlson, \& Salamone, 2001). Interestingly, the receptor mechanisms through which DA may be acting in the PFC to bias decision making are slightly different from those in the ventral striatum. A pharmacological blockade of $\mathrm{D}_{1}$ - but not of $\mathrm{D}_{2}$-receptors in the anterior cingulate reduces the preference for rats to climb over a scalable barrier to obtain a larger (four-pellet) reward (Schweimer \& Hauber, 2006). It is also interesting that the effects of systemic DA antagonism on choice of larger rewards that come with a greater effort (or delay) does not appear to be related to motivational factors. Reducing motivation by providing ad lib access to food does not alter the pattern of choice, even though these manipulations do increase response latencies (Cardinal et al., 2000; Floresco, Tse, \& Ghods-Sharifi, 2008). This finding further supports the notion that DA transmission does not play a specific role in mediating the primary motivational impact of natural reinforcers, such as food. Thus, interference with DA transmission does not appear to induce anhedonia, but, rather, induces anergia, whereby animals are less likely to exert more effort to obtain better rewards. In essence, DA activity helps an organism get to the better things in life, facilitating its ability to overcome effort costs associated with larger or more palatable rewards.

It is important that one consider that while an animal is exerting some type of physical effort to obtain a larger or more palatable reward, it is also incurring an inherent delay to that reward. That is, pressing a lever multiple times or climbing over a barrier delays the retrieval of a reward as compared with the time it takes to consume freely available lab chow or enter an arm that does not have a barrier. With this in mind, it is notable that systemic administration of DA antagonists reduces the preference for larger, delayed rewards in a manner similar to their effect on effort-based tasks (Cardinal et al., 2000; van Gaalen et al., 2006). This begs the question of whether the effects 
of DA manipulations on effort-based decision making are due to alterations of cost-benefit evaluations of how much effort is required to obtain a "better" reward, or whether these effects are due to a reduced tolerance for delayed rewards. To address this question directly, a novel effortdiscounting procedure was devised that allows for manipulations that can tease apart the delay and effort components associated with these types of decisions (Floresco, Tse, \& Ghods-Sharifi, 2008). This task is conducted in an operant chamber in a manner similar to delay-discounting tasks described in the preceding section. Here, rats choose between responding on either an LR lever that delivers two reward pellets after one press, or an HR lever that delivers four reward pellets. However, in order to obtain the HR,

Effort Discounting
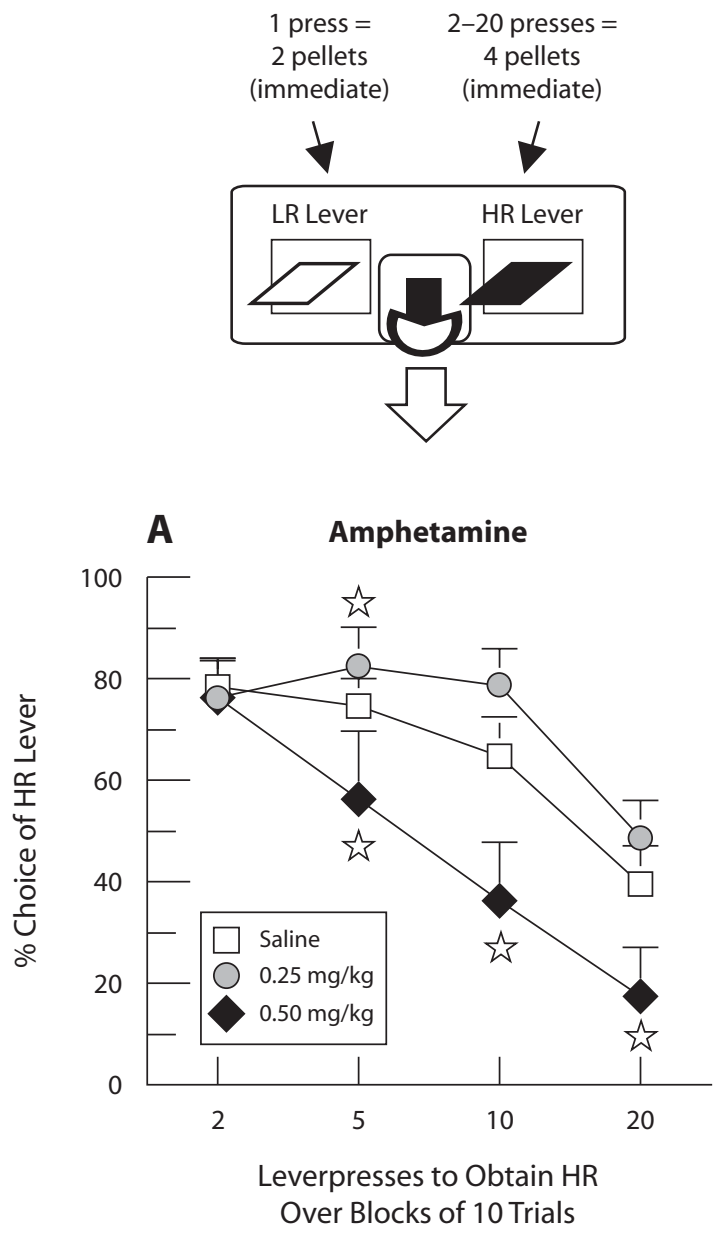

the rat must press the respective lever $2,5,10$, or 20 times, with this ratio increasing over four blocks of discrete trials. With these procedures, the systemic blockade of DA receptors with the broad-spectrum antagonist flupenthixol increased effort discounting, with rats showing a decreased preference for the HR lever. Another experiment investigated the effects of increasing DA transmission with amphetamine, since these manipulations have been shown to increase preferences for larger, delayed rewards using delay-discounting procedures. A relatively low dose of amphetamine $(0.25 \mathrm{mg} / \mathrm{kg})$ increased the tendency for rats to work harder to obtain the HR. However, at a slightly higher dose $(0.50 \mathrm{mg} / \mathrm{kg})$, amphetamine caused a drastic decrease in the preference for the HR lever (Figure 1A).
Effort Discounting

With Equivalent Delays

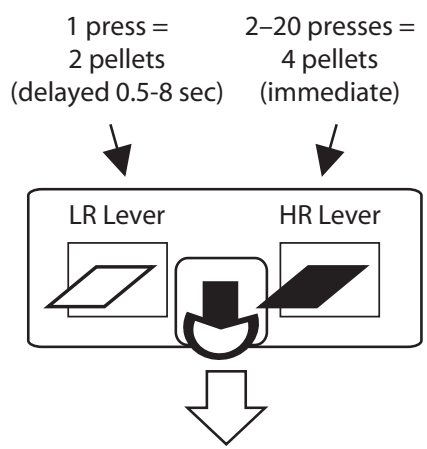

Flupenthixol (DA Antagonist)

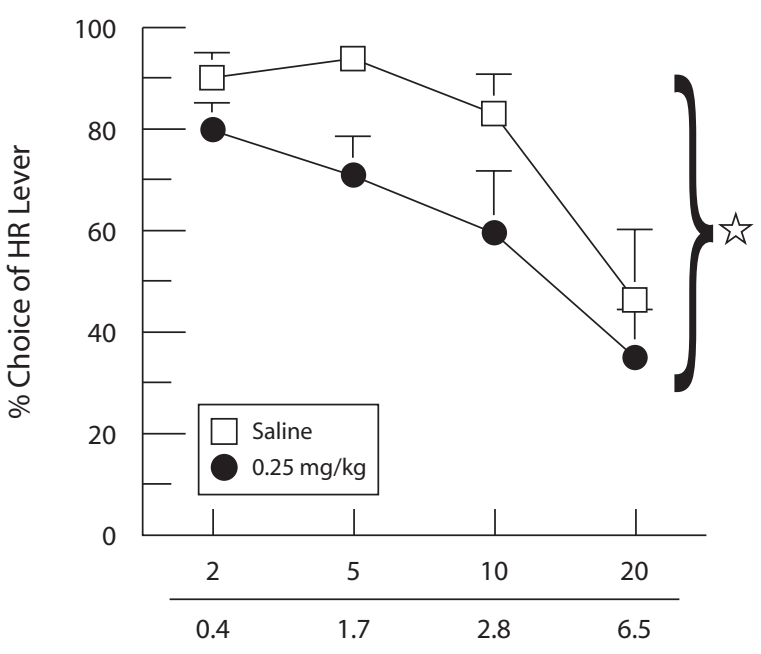

Leverpresses to Obtain HR

Delay to the LR (sec)

Figure 1. (A) Systemic administration of the DA releaser amphetamine exerted differential effects on an effort-discounting task conducted in an operant chamber $(n=8)$. A higher dose $(0.50 \mathrm{mg} / \mathrm{kg})$ decreased the proportion of choices of the HR lever on the effort-discounting task, whereas lower doses $(0.25,0.125 \mathrm{mg} / \mathrm{kg}) \mathrm{had}$ the opposite effect. Stars denote $p<.05$ versus saline. (B) DA receptor blockade with flupenthixol $(0.25 \mathrm{mg} / \mathrm{kg})$ reduced the preference for the HR lever on the effort-discounting with equivalent delays task $(n=8)$. Here, a single press on the LR lever delivered 2 pellets after a delay equivalent to the time required to complete the ratio of presses on the HR lever. Numbers on the abscissa denote the effort requirement on the HR lever (top) and the delay to delivery of the 2 pellets after a single press on the LR lever (bottom). Adapted from Floresco, Tse, \& Ghods-Sharifi (2008). 
As was noted previously, pressing the HR lever repeatedly imposes a considerable delay from the time the initial choice is made to the time the required number of presses is complete, typically ranging from 0.5 to $7 \mathrm{sec}$ on this task. An equivalent delay variation of this task can be used to parse out the relative delay cost associated with the more effortful response. As in the effortdiscounting task, rats were required to press the HR lever multiple times to obtain the four-pellet reward, the delivery of which occurred immediately after the last press. A single press of the LR lever delivered two pellets, but only after a delay equivalent to the time it took for rats to complete the ratio of presses on the HR lever. Thus, if a rat required $7 \mathrm{sec}$ to emit 20 presses on the HR lever to obtain four pellets, then a single press on the LR lever delivered two pellets after a delay of $7 \mathrm{sec}$. This manipulation effectively cancels out the delay cost associated with larger rewards, and it assesses how dopaminergic manipulations may affect cost-benefit decisions related specifically to effort. Under these conditions, the blockade of DA receptors again reduced the preference for a larger reward that came with a greater response cost, directly demonstrating that DA antagonists affect effortrelated decisions independent of their effects on delay discounting (Figure 1B). Furthermore, the increased preference for the HR lever induced by a lower dose of amphetamine was no longer apparent when rats were tested using the equivalent delay procedure, suggesting that the effects of this dose were attributable primarily to an increased tolerance for delayed rewards. However, the "laziness" induced by a higher dose of amphetamine was still apparent when rats were tested using this modified procedure, suggesting that abnormal increases in DA activity can hamper cost-benefit evaluations related to effort. Again, it is interesting that similar doses of amphetamine have been shown to increase preference for larger delayed rewards (Cardinal et al., 2000; van Gaalen et al., 2006). This suggests that psychostimulant drugs, such as amphetamine, can exert differential effects on certain forms of cost-benefit decision making, depending on the particular type of costs animals must evaluate. Furthermore, the fact that either increases or decreases in DA activity can alter this form of decision making in the same manner is in keeping with the notion that dopaminergic modulation of certain executive functions takes the form of an inverted U-shaped curve, where too little or too much DA activity can impair performance (Floresco \& Magyar, 2006). Preliminary studies have since confirmed that the inactivation of the NAc core (but not the shell) induces an effect similar to that of systemic DA antagonists on this task, reducing the preference of the HR lever under normal conditions, and using the equivalent delay procedures (Ghods-Sharifi \& Floresco, 2007). Thus, these studies show that the NAc and DA transmission in this nucleus play a critical role in enabling animals to overcome effort-related costs associated with larger or more palatable rewards - a function independent of their role in delay-based decision making.

\section{Risk-Based Decision Making}

Prefrontal cortex. As opposed to delay or effort discounting, relatively few studies have investigated the neural basis of risk-based decision making in animals. Of these, the OFC has been the region receiving the most attention. In one study, Mobini and colleagues (2002) assessed the effects of lesions to the OFC on the sensitivity to probabilistic reinforcement. In this study, female rats that had received lesions to the OFC were trained on a probabilistic discounting task conducted in an operant chamber. A daily training session consisted of 50 forced choices, followed by 10 discrete free choice trials in which rats chose between two levers: one that delivered 1 pellet immediately, and another that delivered 2 pellets in a probabilistic manner. The probability of obtaining the larger reward remained constant throughout a daily session; however, the odds of receiving the larger reward were decreased systematically every $20-25$ days. Rats with lesions of the OFC displayed a reduced preference for larger, probabilistic rewards than did the sham operated controls - a result that on first glance suggests that these manipulations may have enhanced risk-averse tendencies. However, these risk-averse tendencies were most prominent when the odds of receiving the larger, 2-pellet reward were either $32 \%$ or $20 \%$. Note that under these conditions, choosing the smaller but certain 1-pellet option would yield a greater payoff over the 10 free choice trials, as compared with the reward that could be obtained if the rats had selected the riskier lever (i.e., 10 pellets vs. $\sim 4-7$ pellets, on average). This result suggests that rats with OFC lesions were more sensitive to the overall amount of reinforcement that may have been obtained over a training session, rather than the amount that could be obtained on each trial. It is also worth noting that because lesions were induced prior to any behavioral training, these manipulations may have affected learning about the relative magnitudes of rewards associated with each lever. Furthermore, in many of the subjects, lesions to the OFC were quite large, encompassing other regions of the medial PFC, making it difficult to confirm which region of the PFC may have been mediating these effects.

More recent studies had investigated the effects of OFC lesions on a choice task involving uncertain (rather than known) probabilities of reward. There is evidence to suggest that there may be distinct differences in the mechanisms underlying decisions involving unknown probabilities of reward (uncertain or ambiguous decision making) and known probabilities of reward (risk-based decision making; Brand et al., 2007; Hsu, Bhatt, Adolphs, Tranel, \& Camerer, 2005). Given that most of the previous research conducted on human patients with OFC damage used tasks that assessed decision making during uncertain/ambiguous conditions (Bechara et al., 1994; Bechara et al., 1999), Pais-Vieira, Lima, and Galhardo (2007) chose to examine how discrete lesions of the OFC would disrupt performance of a similar decision-making task in rats, in order to compare the results of animal and human experiments. Rats were trained preoperatively to press levers for a food reward, and they had experience 
with choices that led to no reward, but they remained naive to the actual decision-making task to which they would be subjected. After receiving excitotoxic lesions of the OFC, they were trained over a single session on a task conducted in a novel testing apparatus. Here, rats chose between two chambers, each of which had a single lever and a foodpellet dispenser. Entering one chamber and pressing the lever delivered one pellet with an $80 \%$ probability (low risk). Choosing the lever in the other chamber delivered three pellets, but only with a $30 \%$ probability (high risk). Thus, this task was similar in some respects to the Iowa Gambling Task, in that rats had no prior knowledge of the consequences of choosing each option and were unaware of the duration of the task. However, unlike other risk-based decision-making tasks, the long-term gain associated with choosing either lever was approximately the same, therefore negating the use of a strategy for optimal choice. OFC-lesioned rats performed similarly to shamlesioned rats during the beginning of the task, showing no preference for either lever. As the session progressed, control rats eventually selected the low-risk option associated with smaller reliable rewards more frequently. In contrast, OFC-lesioned rats switched their preference to larger, unreliable rewards. These lesions also reduced sensitivity to risk assessment; these animals persisted in choosing the high-risk lever regardless of the success of their choice on the previous trial. These results are contrary to those found by Mobini et al. (2002), who reported that OFC lesions induced risk aversion. It is plausible that the differences between these two studies were attributable to the differences in behavioral protocols. In the study by Mobini et al. (2002), rats learned the probabilities associated with larger rewards over extended training, whereas in the protocol used by Pais-Vieira et al. (2007), rats chose under conditions of ambiguity, and they learned the odds and rewards associated with each choice in a single training session. Note that there have been similar discrepancies in the human literature with regard to the effects of OFC lesions on risk-based decision making (Bechara et al., 1994; Rogers et al., 1999). Thus, it is apparent that in both rats and humans, the effects of OFC lesions can be quite variable, depending on the type of test used to assess this risk-based decision making and on whether subjects are provided with information a priori about the relative risks associated with each choice. Moreover, the contribution that the more medial regions of the PFC make to these processes remains to be explored.

Amygdala. As was noted previously, the amygdala is a critical structure for processing emotionally salient or arousing stimuli, and it has been shown to be associated with anticipating rewards and punishments (Phelps \& LeDoux, 2005). It is somewhat surprising that-to our knowledge - there have been no studies examining the role of the amygdala in risk-based decision making using animal paradigms. As was described previously, when animals choose between a small, immediate reward and a large, delayed reward, basolateral amygdala lesions increase impulsivity by decreasing the preference for the large, delayed reward as the length of the delay increases (Winstanley et al., 2004). A similar effect has been observed with effort-based decision making, where bilateral inactivations of the amygdala also decrease the preference for the large reward associated with a greater amount of work (Floresco \& Ghods-Sharifi, 2007). These findings suggest that the amygdala may bias choice toward larger rewards that come with a greater cost, and that lesions of this structure shift preference to smaller, but cheaper, rewards. Yet, patients with amygdala damage display an increased preference for larger but riskier rewards (Bechara et al., 1999; Brand et al., 2007; Weller et al., 2007). It is noteworthy that the risky patterns of choice observed in amygdala patients ultimately yield less overall reward in the long term. Likewise, animals with lesions of the amygdala opt for smaller rewards that come with a lesser cost, which also yields less overall reward over repeated trials (Floresco \& Ghods-Sharifi, 2007; Winstanley et al., 2004). Thus, it is possible that lesions of the amygdala interfere with processes that bias choice behavior toward options that will lead to greater long-term payoffs, which - depending on the task at hand-would induce impulsive, lazy, or risky patterns of choice. As such, it would be of particular interest to assess the effects of amygdala lesions (particularly the basolateral nucleus, which projects to the PFC and NAc) on risk-based decision making in animals.

Nucleus accumbens. Using a choice paradigm adapted from delay-discounting tasks described previously (Cardinal et al., 2000), Cardinal and Howes (2005) examined the effects of lesions of the more lateral core region of the nucleus accumbens on the preference for a risky/large reward. In a discrete-trial task, rats chose between a single food pellet delivered with $100 \%$ certainty versus four pellets delivered with a range of probabilities of reward (ranging from $100 \%$ to $6.25 \%$ ). However, unlike other tasks used to assess risk-based decision making in rodents, the probability of obtaining the larger reward changed systematically over blocks of trials throughout the course of daily training sessions. After an extended period of preoperative training, rats received excitotoxic or sham lesions of the NAc core and then retrained on the task. In line with optimal choice, the proportion of choices of the large/risky lever made by control rats decreased monotonically as the probability of reward decreased throughout a session. Rats that had received lesions of the NAc core displayed a risk-averse pattern of choice behavior. After 20 retraining sessions, these rats displayed stable levels of choice in which they chose the risky/large reinforcer significantly less often than did sham-lesioned rats. In this task, the optimal indifference point (i.e., the point at which they should select both options with equal frequency) was when the probability of receiving a larger reward was $25 \%$. Yet, after extended training, lesioned rats displayed an indifference point of $70 \%$. These effects were maintained regardless of whether the probability of the risky/large reinforcer decreased or increased over the course of a session, and whether the animals were food deprived or sated.

In another study, large lesions of the NAc incorporating both core and shell regions did not induce a statisti- 
cally significant difference in choice behavior using an adjusting-amount procedure in which rats chose between either a standard amount of water reward delivered with a fixed probability or an immediate reward, the magnitude of which changed over the course of a training session (Acheson et al., 2006). However, there was a trend toward risk aversion for the lesioned animals. Thus, it is possible that tasks requiring adjustments in behavior in response to changes in the relative risks of obtaining larger rewards may be more sensitive to lesions of the NAc core. Despite the differences in these studies, the fact that lesions of this nucleus induce risk aversion complements findings from functional imaging studies in which increased activation of the ventral striatum is associated with risky choices (Kuhnen \& Knutson, 2005). It is notable that the role of the medial shell region of the NAc in this form of decision making has not been investigated directly. Given that the core and shell have been shown to play dissociable roles in other functions mediated by the PFC (Floresco, Ghods-Sharifi, Vexelman, \& Magyar, 2006; Floresco, McLaughlin, \& Haluk, 2008), it would be of great interest to compare the contributions that these two adjacent regions of the ventral striatum make to risk-based judgments. Nevertheless, these studies suggest that intact NAc functioning biases the direction of an individual choice toward potentially larger rewards, even though they may come with a greater risk.

Dopamine. At the neurophysiological level, recordings of midbrain DA neurons from awake behaving monkeys have identified neurons that respond to reward probability (Fiorillo, Tobler, \& Schultz, 2003). The presentation of reward-related stimuli induces a gradual increase in the firing of these neurons prior to the potential delivery of a reward. However, stimuli associated with the greatest amount of uncertainty about reward delivery (i.e., those that are predictive $50 \%$ of the time) induce much larger increases in DA neuron activity as compared with what is observed when the probability of reward delivery is certain (either $100 \%$ or $0 \%$ ). Therefore, midbrain DA neurons may be encoding information about reward uncertainty, with the greatest increase in DA activity being associated with conditions in which reward delivery is most uncertain.

Although there has a been a substantial amount of psychopharmacological research investigating the contribution of DA in modulating other types of cost-benefit decision making related to delay or effort (Cardinal et al., 2000; Floresco, Tse, \& Ghods-Sharifi, 2008; van Gaalen et al., 2006), it is somewhat surprising that there is a relative paucity of similar studies looking at risk-based decision making. Kaminksi and Ator (2001) examined the effects of amphetamine on a probabilistic choice task in which rats chose between either a certain lever that delivered 3 pellets or a risky lever that produced 15 pellets, but with a probability of $33 \%$. Systemic administration of amphetamine resulted in differential effects on risky choice, depending on the duration of the intertrial interval and baseline levels of risky choice of an individual rat. However, some of the doses used in this study $(1.8-5.6 \mathrm{mg} / \mathrm{kg})$ were substantially higher than those used in other studies examining the effects of amphetamine in delay and effort discounting $(0.25-1.0 \mathrm{mg} / \mathrm{kg})$. These higher doses typically induce stereotypy, making interpretation of these data problematic.

Recent work has begun to investigate the role of DA in the mediation of risky choice in animals, using a variation of the probabilistic discounting task that was described by Cardinal and Howes (2005), in which hungry rats are forced to choose between one of two response options in an operant chamber. One lever delivers a smaller reward (one pellet) with $100 \%$ probability, whereas the other may deliver a larger reward (four pellets) with a probability that decreases over a training session $(100 \%, 50 \%, 25 \%$, or $12.5 \%$; Figure $2 \mathrm{~A}$ ). These preliminary experiments assessed the effects of systemic dopaminergic manipulations on risky choice using animals that had been previously trained on an effort-discounting task, enabling a direct comparison of the effects these manipulations had on both forms of decision making in the same animals (Ghods-Sharifi, St. Onge, \& Floresco, 2006).

As can be observed in Figure 2B, under control conditions (saline), rats adopt a near-optimal choice strategy that changes over the training session. After choosing the large-risky lever on almost every trial during the first block $(100 \%)$, rats alter their decision making, choosing the risky lever less often over subsequent blocks as the probability of reinforcement decreases. Interestingly, on the final block $(12.5 \%)$, optimal foraging strategy dictates choosing the small-certain lever every trial, since this would yield more reinforcement (10 pellets) than the large-risky lever (4 or possibly 8 pellets). Yet, they still choose the risky option on $30 \%-40 \%$ of trials. The blockade of DA receptors with the broad-spectrum DA antagonist flupenthixol decreased in the proportion of choices directed toward the large-risky lever (Figure 2B, black circles), as compared with the pattern of choice displayed by the same rats following control treatments. The riskaverse pattern of choice induced by DA receptor blockade complements findings from studies in humans, in which reducing DA synthesis via acute phenylalanine and tyrosine depletion induces a robust reduction in risk-taking behavior on the Cambridge Gambling Task in patients who are recovered from depression (Roiser et al., 2005). Recall that the administration of this or other DA antagonists also reduces the preference for larger rewards that either are delayed (Floresco, Tse, \& Ghods-Sharifi, 2008; van Gaalen et al., 2006) or require more effort (Floresco, Tse, \& Ghods-Sharifi, 2008). In contrast, increasing DA transmission with a relatively low dose of amphetamine $(0.5 \mathrm{mg} / \mathrm{kg})$ had the opposite effect, inducing a dramatic increase in risky choice (Figure 2B, black diamonds). This was a particularly interesting finding, considering that in the same animals, similar treatments reduced the preference for larger rewards associated with a greater effort cost (Figure 1A, Floresco, Tse, \& Ghods-Sharifi, 2008). Moreover, the increase in risky choice induced by enhanced DA activity draws a striking parallel to clinical studies in which DA agonist treatments have been associ- 
A

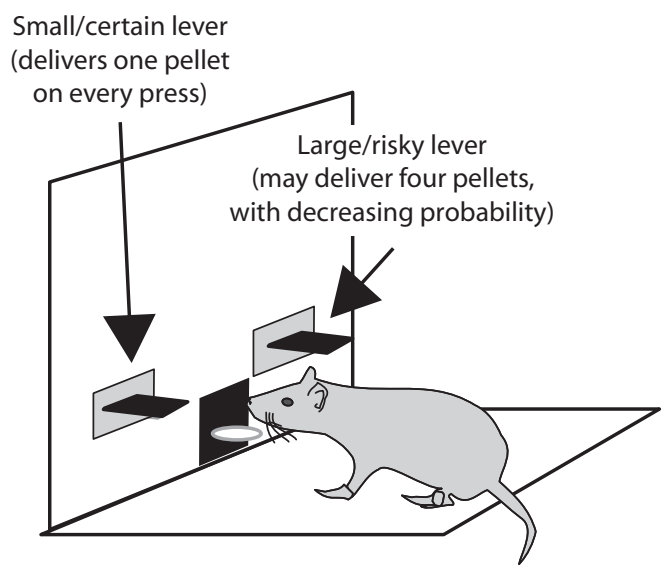

B

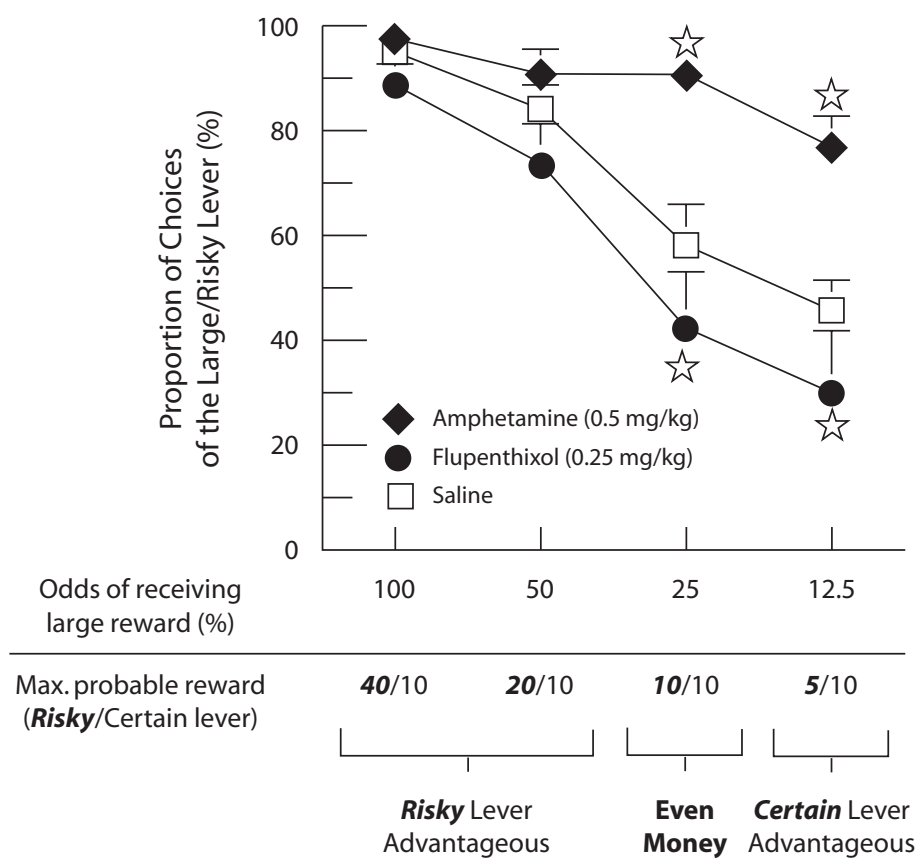

Figure 2. (A) Diagram of the risk-discounting task. Over discrete trials, rats choose to respond on either the certain/small lever that delivers one pellet on every press, or the large/risky lever, that may or may not deliver four pellets. (B) Proportion of choices made on the large/risky lever during free-choice trials across the four blocks of the risk-discounting task $(n=8$; Ghods-Sharifi et al., 2006). Analysis of these data revealed a significant drug treatment $\times$ trial block interaction $[F(6,42)=5.27, p<.01]$. Under control conditions, rats choose the risky lever less as the probability of obtaining a large reward decrease. The blockade of DA receptors with flupenthixol induces risk aversion. In contrast, amphetamine $(0.5 \mathrm{mg} / \mathrm{kg})$ significantly increases risky choice. Stars denote $p<.05$ versus saline treatments. The $x$-axis denotes the odds of receiving the large reinforcer following a response on a risky lever over the four trial blocks (top), and the maximal average reward that could be obtained in each block if the animal responded exclusively on the risky or certain lever (bottom).

ated with an increase in pathological gambling tendencies in a subpopulation of patients (Quickfall \& Suchowersky, 2007; Seedat, Kesler, Niehaus, \& Stein, 2000; Voon et al., 2006). These findings indicate that increases or decreases in DA transmission may hamper calculations about the relative risks associated with a particular choice, resulting in an over- or underestimation of the likelihood of obtaining rewards delivered in a probabilistic manner. The brain regions in which DA may be acting to affect this form of decision making - and the specific receptor subtypes that may be involved-merit further investigation.

\section{Summary}

When viewed collectively, these studies in experimental animals provide novel insight into the contributions made by anatomically distinct nodes in cortical, limbic, and striatal circuitry to different types of cost-benefit decision making. As has been observed in humans, different regions of the medial and orbital PFC modulate risk, delay, and effort discounting. However, it appears that anatomically distinct regions of the frontal lobes subserve specialized functions, each of which may contribute to evaluations of a specific type of response cost. The amygdala, working in conjunction with the PFC, also makes an important contribution to decision making and may facilitate an organism's ability to overcome a variety of costs to maximize long-term benefits. Likewise, the NAc appears to play a fundamental biasing role, permitting information about the relative costs and benefits associated with particular choices (processed by corticolimbic circuits) to promote actions that may yield greater payoffs. In essence, the NAc - in cooperation with the DA system-helps an organism to overcome environmental obstacles (i.e., time, work, risk) that could hinder the selection of actions that normally yield more favorable outcomes. Furthermore, systemic decreases in DA activity appear to exert a similar effect on different types of decision making, whereas abnormal increases in this activity can have differential effects on behavior, dependent in part on the specific type of costbenefit appraisals an organism may be faced with. The observation that similar neural circuits are recruited to resolve cost-benefit decisions in both humans and animals is encouraging, since it suggests that animal models of decision making will make an important contribution to our understanding of these processes. Since impaired decision making is a prominent symptom associated with a variety of neuropsychiatric disorders, it is likely that preclinical studies of this kind will provide further insight into the pathophysiological processes that contribute to the form of executive dysfunction observed in these diseases. 


\section{AUTHOR NOTE}

Some of the studies reviewed in this article were supported by grants from the Canadian Institutes of Health Research (CIHR), the Natural Sciences and Engineering Research Council of Canada, and Parkinson's Society Canada. J.R.S.O. is the recipient of a Michael Smith Graduate Student Fellowship, and S.B.F. is a CIHR New Investigator and a Michael Smith Scholar. Address correspondence to S. B. Floresco, Department of Psychology and Brain Research Centre, University of British Columbia, 2136 West Mall, Vancouver, BC, V6T 1 Z4 Canada (e-mail: floresco@psych.ubc.ca).

\section{REFERENCES}

Acheson, A., Farrar, A. M., Patak, M., Hausknecht, K. A., Kieres, A. K., CHOI, S., ET AL. (2006). Nucleus accumbens lesions decrease sensitivity to rapid changes in the delay to reinforcement. Behavioural Brain Research, 173, 217-228.

Balleine, B. W., \& Dickinson, A. (1998). Goal-directed instrumental action: Contingency and incentive learning and their cortical substrates. Neuropharmacology, 37, 407-419.

Barch, D. M., Braver, T. S., AKbudak, E., Conturo, T., Ollinger, J., \& SNYDER, A. (2001). Anterior cingulate cortex and response conflict: Effects of response modality and processing domain. Cerebral Cortex, 11, 837-848.

Barch, D. M., Braver, T. S., Nystrom, L. E., Forman, S. D., Noll, D. C., \& CoHEN, J. D. (1997). Dissociating working memory from task difficulty in human prefrontal cortex. Neuropsychologia, 35, 1373-1380.

Baxter, M. G., Parker, A., Lindner, C. C., Izquierdo, A. D., \& MurRAY, E. A. (2000). Control of response selection by reinforcer value requires interaction of amygdala and orbital prefrontal cortex. Journal of Neuroscience, 20, 4311-4319.

Bechara, A., Damasio, A. R., Damasio, H., \& Anderson, S. W. (1994). Insensitivity to future consequences following damage to human prefrontal cortex. Cognition, 50, 7-15.

Bechara, A., Damasio, H., Damasio, A. R., \& Lee, G. P. (1999). Different contributions of the human amygdala and ventromedial prefrontal cortex to decision-making. Journal of Neuroscience, 19, 5473-5481.

Bechara, A., Damasio, H., Tranel, D., \& Anderson, S. W. (1998). Dissociation of working memory from decision making within the human prefontal cortex. Journal of Neuroscience, 18, 428-437.

Bizot, J. C., Le Bihan, C., Puech, A. J., Hamon, M., \& Thiebot, M. H. (1999). Serotonin and tolerance to delay of reward in rats. Psychopharmacology, 146, 400-412.

Blair, K., Marsh, A. A., Morton, J., Vythilingam, M., Jones, M., Mondillo, K., ET AL. (2006). Choosing the lesser of two evils, the better of two goods: Specifying the roles of ventromedial prefrontal cortex and dorsal anterior cingulate in object choice. Journal of Neuroscience, 26, 11379-11386.

Boulougouris, V., Dalley, J. W., \& Robbins, T. W. (2007). Effects of orbitofrontal, infralimbic and prelimbic cortical lesions on serial spatial reversal learning in the rat. Behavioural Brain Research, 179, 219-228.

Brand, M., Grabenhorst, F., Starcke, K., Vandekerckhove, M. M., \& Markowitsch, H. J. (2007). Role of the amygdala in decisions under ambiguity and decisions under risk: Evidence from patients with Urbach-Wiethe disease. Neuropsychologia, 45, 1305-1317.

Cardinal, R. N., Daw, N., Robbins, T. W., \& Everitt, B. J. (2002). Local analysis of behavior in the adjusting-delay task for assessing choice of delayed reinforcement. Neural Networks, 15, 617-634.

Cardinal, R. N., \& Howes, N. J. (2005). Effects of lesions of the nucleus accumbens core on choice between small certain rewards and large uncertain rewards in rats. BMC Neuroscience, 6, 37.

Cardinal, R. N., Parkinson, J. A., Marbini, H. D., Toner, A. J., Bussey, T. J., Robbins, T. W., \& Everitt, B. J. (2003). Role of the anterior cingulate cortex in the control over behavior by Pavlovian conditioned stimuli in rats. Behavioral Neuroscience, 117, 566-587.

Cardinal, R. N., Pennicott, D. R., Sugathapala, C. L., Robbins, T. W., \& EveritT, B. J. (2001). Impulsive choice induced in rats by lesions of the nucleus accumbens core. Science, 292, 2499-2501.

Cardinal, R. N., Robbins, T. W., \& Everitt, B. J. (2000). The effects of d-amphetamine, chlordiazepoxide, alpha-flupenthixol and behav- ioural manipulations on choice of signalled and unsignalled delayed reinforcement in rats. Psychopharmacology, 152, 362-375.

Chudasama, Y., Passetti, F., Rhodes, S. E. V., Lopian, D., Desai, A., $\&$ RobBins, T. W. (2003). Dissociable aspects of performance on the 5-choice serial reaction time task following lesions of the dorsal anterior cingulate, infralimbic and orbitofrontal cortex in the rat: Differential effects on selectivity, impulsivity and compulsivity. Behavioural Brain Research, 146, 105-119.

Chudasama, Y., \& Robbins, T. W. (2003). Dissociable contributions of the orbitofrontal and infralimbic cortex to Pavlovian autoshaping and discrimination reversal learning: Further evidence for the functional heterogeneity of the rodent frontal cortex. Journal of Neuroscience, 23, 8771-8780.

Clark, L., Manes, F., Antoun, N., Sahakian, B. J., \& Robbins, T. W. (2003). The contributions of lesion laterality and lesion volume to decision-making impairment following frontal lobe damage. Neuropsychologia, 41, 1474-1483.

Cole, B. J., \& Robbins, T. W. (1989). Effects of 6-Hydroxydopamine lesions of the nucleus accumbens septi on performance of a 5-choice serial reaction time task in rats-Implications for theories of selective attention and arousal. Behavioural Brain Research, 33, 165-179.

Cools, R., Barker, R. A., Sahakian, B. J., \& Robbins, T. W. (2003). L-Dopa medication remediates cognitive inflexibility, but increases impulsivity in patients with Parkinson's disease. Neuropsychologia, 41, 1431-1441.

Corbit, L. H., \& Balleine, B. W. (2005). Double dissociation of basolateral and central amygdala lesions on the general and outcomespecific forms of Pavlovian-instrumental transfer. Journal of Neuroscience, 25, 962-970.

Cousins, M. S., Wei, W., \& Salamone, J. D. (1994). Pharmacological characterization of performance on a concurrent lever pressing/ feeding choice procedure: Effects of dopamine antagonist, cholinomimetic, sedative and stimulant drugs. Psychopharmacology, 116, 529-537.

Dalley, J. W., Cardinal, R. N., \& Robbins, T. W. (2004). Prefrontal executive and cognitive functions in rodents: Neural and neurochemical substrates. Neuroscience \& Biobehavioral Reviews, 28, 771-784.

Dietrich, A., \& Allen, J. D. (1998). Functional dissociation of the prefrontal cortex and the hippocampus in timing behavior. Behavioral Neuroscience, 112, 1043-1047.

Evenden, J. L., \& RyAn, C. N. (1996). The pharmacology of impulsive behavior in rats: The effects of drugs on response choice with varying delays of reinforcement. Psychopharmacology, 128, 161-170.

Fellows, L. K. (2007). The role of orbitofrontal cortex in decision making: A component process account. In G. Schoenbaum, J. A. Gottfried, E. A. Murray, \& S. J. Ramus (Eds.), Linking affect to action: Critical contributions of the orbitofrontal cortex (Annals of the New York Academy of Sciences, Vol. 1121, pp. 421-430). New York: New York Academy of Sciences.

Fellows, L. K., \& FARAH, M. J. (2005). Different underlying impairments in decision-making following ventromedial and dorsolateral frontal lobe damage in humans. Cerebral Cortex, 15, 58-63.

Fiorillo, C. D., Tobler, P. N., \& Schultz, W. (2003). Discrete coding of reward probability and uncertainty by dopamine neurons. Science, 299, 1898-1902.

Floresco, S. B. (2007). Dopaminergic regulation of limbic-striatal interplay. Journal of Psychiatry \& Neuroscience, 32, 400-411.

Floresco, S. B., \& GHods-Sharifi, S. (2007). Amygdala-prefrontal cortical circuitry regulates effort-based decision making. Cerebral Cortex, 17, 251-260.

Floresco, S. B., Ghods-Sharifi, S., Vexelman, C., \& Magyar, O. (2006). Dissociable roles for the nucleus accumbens core and shell in regulating set shifting. Journal of Neuroscience, 26, 2449-2457.

Floresco, S. B., \& MAGYAR, O. (2006). Mesocortical dopamine modulation of executive functions: Beyond working memory. Psychopharmacology, 188, 567-585.

Floresco, S. B., Mclaughlin, R. J., \& Haluk, D. M. (2008). Opposing roles for the nucleus accumbens core and shell in cue-induced reinstatement of food-seeking behavior. Neuroscience, 154, 877-884.

Floresco, S. B., Tse, M. T. L., \& Ghods-Sharifi, S. (2008). Dopaminergic and glutamatergic regulation of effort- and delay-based decision making. Neuropsychopharmacology, 33, 1966-1979. 
Fu, C. H., Morgan, K., Suckling, J., Williams, S. C., Andrew, C., Vythelingum, G. N., \& McGuire, P. K. (2002). A functional magnetic resonance imaging study of overt letter verbal fluency using a clustered acquisition sequence: Greater anterior cingulate activation with increased task demand. NeuroImage, 17, 871-879.

Garrud, P., Goodall, G., \& Mackintosh, N. J. (1981). Overshadowing of a stimulus-reinforcer association by an instrumental response. Quarterly Journal of Experimental Psychology, 33B, 123-135.

Ghods-Sharifi, S., \& Floresco, S. B. (2007). Dissociable involvement of nucleus accumbens subregions in effort-based decision making. Society for Neuroscience Abstracts, 741.2.

Ghods-Sharifi, S., St. Onge, J. R., \& Floresco, S. B. (2006). Dopaminergic modulation of effort and risk-based decision making. Neuropsychopharmacology, 31, S207.

Hariri, A. R., Brown, S. M., Williamson, D. E., Flory, J. D., DE Wit, H., \& MANUCK, S. B. (2006). Preference for immediate over delayed rewards is associated with magnitude of ventral striatal activity. Journal of Neuroscience, 26, 13213-13217.

Heerey, E. A., Robinson, B. M., McMahon, R. P., \& Gold, J. M. (2007). Delay discounting in schizophrenia. Cognitive Neuropsychiatry, 12, 213-221.

Hsu, M., Bhatt, M., Adolphs, R., Tranel, D., \& Camerer, C. F. (2005). Neural systems responding to degrees of uncertainty in human decision-making. Science, 310, 1680-1683.

Hutton, S. B., Murphy, F. C., Joyce, E. M., Rogers, R. D., CuthBERT, I., BARNES, T. R., ET AL. (2002). Decision making deficits in patients with first-episode and chronic schizophrenia. Schizophrenia Research, 55, 249-257.

KAMINSKI, B. J., \& AtoR, N. A. (2001). Behavioral and pharmacological variables affecting risky choice in rats. Journal of the Experimental Analysis of Behavior, 75, 275-297.

Kelly, P. H., Seviour, P. W., \& Iversen, S. D. (1975). Amphetamine and apomorphine responses in the rat following 6-OHDA lesions of the nucleus accumbens septi and corpus striatum. Brain Research, 94, 507-522.

Kheramin, S., Body, S., Ho, M. Y., Velazquez-Martinez, D. N., Bradshaw, C. M., Szabadi, E., ET AL. (2004). Effects of orbital prefrontal cortex dopamine depletion on intertemporal choice: A quantitative analysis. Psychopharmacology, 175, 206-214.

Koов, G., Riley, S. T., Smith, S. C., \& Robins, T. W. (1978). Effects of 6-hydroxydopamine lesions of the nucleus accumbens septi and olfactory tubercle on feeding, locomotor activity and amphetamine anorexia in the rat. Journal of Comparative Physiology \& Psychology, 92, 917-927.

Kunnen, C. M., \& Knutson, B. (2005). The neural basis of financial risk taking. Neuron, 47, 763-770.

Lattal, K. A., \& Gleeson, S. (1990). Response acquisition with delayed reinforcement. Journal of Experimental Psychology: Animal Behavioral Processes, 16, 27-39.

Manes, F., Sahakian, B., Clark, L., Rogers, R., Antoun, N., AitKEN, M., \& RobBIns, T. (2002). Decision-making processes following damage to the prefrontal cortex. Brain, 125, 624-639.

Marsh, A. A., Blair, K. S., Vythilingam, M., Busis, S., \& Blair, R. J. (2007). Response options and expectations of reward in decisionmaking: The differential roles of dorsal and rostral anterior cingulate cortex. NeuroImage, 35, 979-988.

MAZUR, J. (1987). An adjusting procedure for studying delayed reinforcement. In M. L. Commons, J. A. Nevin, \& H. C. Rachlin (Eds.), Quantitative analyses of behavior: The effect of delay and intervening events on reinforcement value (pp. 55-73). Hillsdale, NJ: Erlbaum.

Mobini, S., Body, S., Ho, M. Y., Bradshaw, C. M., Szabadi, E., DEAKIN, J. F., \& ANDERSON, I. M. (2002). Effects of lesions of the orbitofrontal cortex on sensitivity to delayed and probabilistic reinforcement. Psychopharmacology, 160, 290-298.

Monterosso, J., Ehrman, R., Napier, K. L., O’Brien, C. P., \& Childress, A. R. (2001). Three decision-making tasks in cocainedependent patients: Do they measure the same construct? Addiction, 96, 1825-1837.

Must, A., Szabó, Z., Bódi, N., SzÁsz, A., JANKA, Z., \& KÉRI, S. (2006). Sensitivity to reward and punishment and the prefrontal cortex in major depression. Journal of Affective Disorders, 90, 209-215.

Naccache, L., Dehaene, S., Cohen, L., Habert, M. O., Guichart-
Gomez, E., Galanaud, D., \& Willer, J. C. (2005). Effortless control: Executive attention and conscious feeling of mental effort are dissociable. Neuropsychologia, 43, 1318-1328.

Nowend, K. L., Arizzi, M., Carlson, B. B., \& Salamone, J. D. (2001). D1 or D2 antagonism in nucleus accumbens core or dorsomedial shell suppresses leverpressing for food but leads to compensatory increases in chow consumption. Pharmacology, Biochemistry, \& Behavior, 69, 373-382.

O'DoherTy, J. P. (2004). Reward representations and reward-related learning in the human brain: Insights from neuroimaging. Current Opinion in Neurobiology, 14, 769-776.

Pagonabarraga, J., García-Sánchez, C., Llebaria, G., PascualSedano, B., Gironell, A., \& Kulisevsky, J. (2007). Controlled study of decision-making and cognitive impairment in Parkinson's disease. Movement Disorders, 22, 1430-1435.

Pais-Vieira, M., Lima, D., \& Galhardo, V. (2007). Orbitofrontal cortex lesions disrupt risk assessment in a novel serial decision-making task for rats. Neuroscience, 145, 225-231.

Paulus, M. P., \& Frank, L. R. (2006). Anterior cingulate activity modulates nonlinear decision weight function of uncertain prospects. NeuroImage, 30, 668-677.

Phelps, E. A., \& LeDoux, J. E. (2005). Contributions of the amygdala to emotion processing: From animal models to human behavior. $\mathrm{Neu}$ ron, 20, 175-187.

Pratt, W. E., \& Mizumori, S. J. Y. (1998). Characteristics of basolateral amygdala neuronal firing on a spatial memory task involving differential reward. Behavioral Neuroscience, 112, 554-570.

Quickfall, J., \& Suchowersky, O. (2007). Pathological gambling associated with dopamine agonist use in restless legs syndrome. Parkinsonism \& Related Disorders, 13, 535-536.

RAGOZZINO, M. E. (2007). The contribution of the medial prefrontal cortex, orbitofrontal cortex, and dorsomedial striatum to behavioral flexibility. In G. Schoenbaum, J. A. Gottfried, E. A. Murray, \& S. J. Ramus (Eds.), Linking affect to action: Critical contributions of the orbitofrontal cortex (Annals of the New York Academy of Sciences, Vol. 1121, pp. 421-430). New York: New York Academy of Sciences.

Reep, R. L., Goodwin, G. S., \& CoRwin, J. V. (1990). Topographic organization in the corticocortical connections of medial agranular cortex in rats. Journal of Comparative Neurology, 294, 262-280.

REYNOLDS, S. M., \& ZAHM, D. S. (2005). Specificity in the projections of prefrontal and insular cortex to ventral striatopallidum and the extended amygdala. Journal of Neuroscience, 25, 11757-11767.

Richards, J. B., Mitchell, S. H., DE Wit, H., \& Seiden, L. S. (1997). Determination of discount functions in rats with an adjusting-amount procedure. Journal of the Experimental Analysis of Behavior, 67, 353-366.

Robiins, T. W., Roberts, D. C., \& Koob, G. (1983). Effects of d-amphetamine and apomorphine upon operant behavior and schedule-induced licking in rats with 6-hydroxydopamine-induced lesions of the nucleus accumbens. Journal of Pharmacology \& Experimental Therapeutics, 224, 662-673.

Rogers, R. D., Everitt, B. J., Baldacchino, A., Blackshaw, A. J., Swainson, R., Wynne, K., ET AL. (1999). Dissociable deficits in the decision-making cognition of chronic amphetamine abusers, opiate abusers, patients with focal damage to prefrontal cortex, and tryptophan-depleted normal volunteers: Evidence for monoaminergic mechanisms. Neuropsychopharmacology, 20, 322-339.

Rogers, R. D., Ramnani, N., Mackay, C., Wilson, J. L., Jezzard, P., CARTER, C. S., \& SMith, S. M. (2004). Distinct portions of anterior cingulate cortex and medial prefrontal cortex are activated by reward processing in separable phases of decision-making cognition. Biological Psychiatry, 15, 594-602.

Roiser, J. P., Mclean, A., Ogilvie, A. D., Blackwell, A. D., BamBER, D. J., GoODYER, I., ET AL. (2005). The subjective and cognitive effects of acute phenylalanine and tyrosine depletion in patients recovered from depression. Neuropsychopharmacology, 30, 775-785.

Rudebeck, P. H., Walton, M. E., Smyth, A. N., Bannerman, D. M., \& Rushworth, M. F. (2006). Separate neural pathways process different decision costs. Nature Neuroscience, 9, 1161-1168.

Saddoris, M. P., Gallagher, M., \& Schoenbaum, G. (2005). Rapid associative encoding in basolateral amygdala depends on connections with orbitofrontal cortex. Neuron, 46, 321-331. 
Salamone, J. D., Correa, M., Farrar, A., \& Mingote, S. M. (2007). Effort-related functions of nucleus accumbens dopamine and associated forebrain circuits. Psychopharmacology, 191, 461-482.

Salamone, J. D., Cousins, M. S., \& Bucher, S. (1994). Anhedonia or anergia? Effects of haloperidol and nucleus accumbens dopamine depletion on instrumental response selection in a T-maze cost/benefit procedure. Behavioural Brain Research, 65, 221-229.

Salamone, J. D., Steinpreis, R. E., McCullough, L. D., Smith, P., Grebel, D., \& Mahan, K. (1991). Haloperidol and nucleus accumbens dopamine depletion suppress leverpressing for food but increase free food consumption in a novel food choice procedure. Psychopharmacology, 104, 515-521.

Salinas, J. A., Packard, M. G., \& McGaugh, J. L. (1993). Amygdala modulates memory for changes in reward magnitude: Reversible posttraining inactivation with lidocaine attenuates the response to a reduction in reward. Behavioural Brain Research, 59, 153-159.

Schoenbaum, G., Chiba, A. A., \& Gallagher, M. (1998). Orbitofrontal cortex and basolateral amygdala encode expected outcomes during learning. Nature Neuroscience, 2, 155-159.

Schoenbaum, G., Chiba, A. A., \& Gallagher, M. (1999). Neural encoding in orbitofrontal cortex and basolateral amygdala during olfactory discrimination learning. Journal of Neuroscience, 19, 1876-1884.

Schoenbaum, G., Setlow, B., \& Ramus, S. J. (2003). A systems approach to orbitofrontal cortex function: Recordings in rat orbitofrontal cortex reveal interactions with different learning systems. Behavioural Brain Research, 146, 19-29.

Schweimer, J., \& Hauber, W. (2005). Involvement of the rat anterior cingulate cortex in control of instrumental responses guided by reward expectancy. Learning \& Memory, 12, 334-342.

Schweimer, J., \& Hauber, W. (2006). Dopamine D1 receptors in the anterior cingulate cortex regulate effort-based decision making. Learning \& Memory, 13, 777-782.

Seedat, S., Kesler, S., Niehaus, D. J., \& Stein, D. J. (2000). Pathological gambling behavior: Emergence secondary to treatment of Parkinson's disease with dopaminergic agents. Depression \& Anxiety, 11, 185-186.

Sesack, S. R., Deutch, A. Y., Roth, R. H., \& Bunney, B. S. (1989). Topographical organization of the efferent projections of the medial prefrontal cortex in the rat: An anterograde tract-tracing study with Phaseolus vulgaris leucoagglutinin. Journal of Comparative Neurology, 290, 213-242.

Shurman, B., Horan, W. P., \& Nuechterlein, K. H. (2005). Schizophrenia patients demonstrate a distinctive pattern of decision-making impairment on the Iowa Gambling Task. Schizophrenia Research, 27, 215-224.

Swainson, R., Rogers, R. D., Sahakian, B. J., Summers, B. A., Polkey, C. E., \& Robbins, T. W. (2000). Probabilistic learning and reversal deficits in patients with Parkinson's disease or frontal or temporal lobe lesions: Possible adverse effects of dopaminergic medication. Neuropsychologia, 38, 596-612.
Tom, S. M., Fox, C. R., Trepel, C., \& Poldrack, R. A. (2007). The neural basis of loss aversion in decision-making under risk. Science, 315, 515-518.

van Gaalen, M. M., van Koten, R., Schoffelmeer, A. N. M., \& VANDERSChUREN, L. J. M. J. (2006). Critical involvement of dopaminergic neurotransmission in impulsive decision making. Biological Psychiatry, 60, 66-73.

Voon, V., Hassan, K., Zurowski, M., Duff-Canning, S., De Souza, M., Fox, S., ET AL. (2006). Prospective prevalence of pathologic gambling and medication association in Parkinson disease. Neurology, 66, 1750-1752.

WADE, T. R., DE WiT, H., \& RichaRds, J. B. (2000). Effects of dopaminergic drugs on delayed reward as a measure of impulsive behavior in rats. Psychopharmacology, 150, 90-101.

Walton, M. E., Bannerman, D. M., Alterescu, K., \& RushWORTH, M. F. (2003). Functional specialization within medial frontal cortex of the anterior cingulate for evaluating effort-related decisions. Journal of Neuroscience, 23, 6475-6479.

Walton, M. E., Bannerman, D. M., \& Rushworth, M. F. (2002). The role of rat medial frontal cortex in effort-based decision making. Journal of Neuroscience, 22, 10996-11003.

Weller, J. A., Levin, I. P., Shiv, B., \& Bechara, A. (2007). Neural correlates of adaptive decision making for risky gains and losses. Psychological Science, 18, 958-964.

Winstanley, C. A., Theobald, D. E., Cardinal, R. N., \& RobBINS, T. W. (2004). Contrasting roles of basolateral amygdala and orbitofrontal cortex in impulsive choice. Journal of Neuroscience, 24, 4718-4722.

Winstanley, C. A., Theobald, D. E., Dalley, J. W., Cardinal, R. N., \& RobBins, T. W. (2006). Double dissociation between serotonergic and dopaminergic modulation of medial prefrontal and orbitofrontal cortex during a test of impulsive choice. Cerebral Cortex, 16, 106114.

Winstanley, C. A., Theobald, D. E., Dalley, J. W., \& Robbins, T. W. (2003). Global 5-HT depletion attenuates the ability of amphetamine to decrease impulsive choice in rats. Psychopharmacology, 170, 320331.

Winstanley, C. A., Theobald, D. E., Dalley, J. W., \& Robbins, T. W. (2005). Interactions between serotonin and dopamine in the control of impulsive choice in rats: Therapeutic implications for impulse control disorders. Neuropsychopharmacology, 30, 669-682.

Wise, R. A., \& Rompre, P. P. (1989). Brain dopamine and reward. Annual Review of Psychology, 40, 191-225.

Zeeb, F. D., Floresco, S. B., \& Winstanley, C. A. (2007). What makes it worth the wait? Investigating the effects of orbitofrontal cortex inactivation on impulsive choice. Society for Neuroscience Abstracts, 741.3 .

(Manuscript received March 21, 2008; revision accepted for publication June 12, 2008.) 